\title{
Coupled RANS/LES for SOFIA Cavity Acoustic Prediction
}

\author{
Stephen Woodruff*
}

\begin{abstract}
A fast but accurate computation is described for the aero-acoustic properties of a large cavity at subsonic flight speeds. This computation employs a detached-eddy simulation model in the free-shear layer at the cavity opening and the surrounding boundary layer, but assumes inviscid flow in the cavity and in the far field. The reduced gridding requirements in the cavity, in particular, lead to dramatic improvements in the time required for the computation. The results of these computations are validated against wind-tunnel data. This approach will permit significantly more flight test points to be evaluated computationally in support of the Stratospheric Observatory For Infrared Astronomy flight-test program being carried out at NASA's Dryden Flight Research Center.
\end{abstract}

\section{Nomenclature}

SOFIA Stratospheric Observatory For Infrared Astronomy

LES Large-Eddy Simulation

RANS Reynolds-Averaged Navier Stokes

DES Detached-Eddy Simulation

$C_{D E S} \quad$ DES coefficient modulating blending between RANS and LES

$u \quad$ Velocity tangential to wall in turbulent boundary layer

$u_{o} \quad$ Velocity tangential to wall outside of boundary layer

$y \quad$ Coordinate normal to wall in boundary layer

$\delta \quad$ Boundary-layer thickness

$n \quad$ Value determining boundary-layer profile a la Prandtl

\section{Introduction}

An effort has been undertaken to provide timely computational cavity aero-acoustic support for the Stratospheric Observatory For Infrared Astronomy (SOFIA) flight-test program at NASA's Dryden Flight Research Center. This effort includes assessing cavity acoustics (including mitigation methods) at operational and non-operational conditions prior to flight tests, rapidly responding to issues raised during the flight-test program and using the computational results to assist with interpretation of the flight-test data.

This is also a valuable opportunity to compare the results of aeroacoustic cavity computations using hybrid LES/RANS techniques against full-scale flight data, and to make improvements in the computational techniques based on those comparisons.

A full assessment of SOFIA cavity acoustics requires computations at many different flight conditions. In addition to the expected operational flight conditions, it is necessary to get data at non-operational flight conditions that might occur in emergencies: the possibility of the cavity door getting stuck at operational altitude, for example, requires checking acoustics at flight conditions all the way down to landing. Performing this assessment requires a reasonably rapid computation of each case. The same is true of the need to respond to analysis needs in a timely manner during flight testing. The weeks such computations have typically taken in the past is too long and so a more rapid approach had to be developed. As described below, this has been accomplished through the use of highly efficient structured-grid codes and by focusing on the significant

\footnotetext{
*Aerospace Engineer, NASA Dryden Flight Research Center, Edwards, CA 93523-0273
} 
physical effects so that computational effort is not wasted on flow features not relevant to the desired results. Extensive wind-tunnel test results are available for detailed validation of this approach $\left(e . g_{.}{ }^{2,3}\right)$.

As the goal of the project is to understand the effect of cavity acoustics on flight safety and mission success, the project is fundamentally concerned with the unsteady aeroacoustics of the cavity and of the aircraft as a whole. Capturing these unsteady processes accurately requires accurate modeling of the unsteady motion of the turbulent shear layer at the cavity opening and thus a hybrid LES/RANS approach is called for: a RANS (Reynolds-Averaged Navier-Stokes) approach would not provide sufficient detail about the unsteady motion and an LES (Large-Eddy Simulation) approach would be far too computationally intensive. Currently, the most commonly used hybrid LES/RANS approach is DES (Detached-Eddy Simulation), which seeks to resolve large-scale eddies in free shear layers explicitly, but relies on RANS modeling for smallerscale boundary-layer turbulence. The DES technique of Strelets, ${ }^{1}$ based on the Mentor SST two-equation turbulence model, is used in the present computations.

Cavity acoustics is an active research topic in a number of areas, including automotive design and aircraft internal weapons bays. The approach developed here should be of value in these areas as well, particularly when deep cavities are involved.

\section{Computational Approach and Grid Development}

Typical computations of cavity acoustic problems have taken several days to several weeks on clusters of four to several dozen processors. ${ }^{4-6}$ The cavity on the SOFIA aircraft is significantly larger than those of earlier studies, which increases the computational complexity of the problem by at least three or four orders of magnitude. The lowest resonance, at $2-3 \mathrm{~Hz}$, is lower by a factor of ten then that of previous studies, requiring that computations be continued for ten times as long. Gridding the larger cavity requires one hundred to one thousand times as many cells, also increasing the computational time. These estimates are borne out in the SOFIA cavity computations of Schmid and colleagues. ${ }^{7-10}$

It would be impossible to meet the goals of the project if the computations took this long. Computations are thus being developed at two levels. The first level involves a computational domain restricted to the cavity and its immediate vicinity, a simplified geometry and flow modeling restricted to only what's necessary to capture the essential physics (DES-modeled turbulent flow in the shear layer, inviscid flow elsewhere). The second level includes the full aircraft and turbulent flow throughout the flow domain. This approach permits rapid computation of cases both before and during flight testing, as well as more detailed computations for validation and addressing specific issues that arise during flight testing. The purpose of the present paper is to report on the development of the first-level computations and their validation with experiment.

Insights gained from SOFIA wind-tunnel studies and the computations of Schmid provide the basis for the simplifying assumptions employed here to make a fast, but accurate, cavity acoustic computation. First, it is noted that test results ${ }^{2,3}$ show the dominant frequencies are below about $200 \mathrm{~Hz}$. This implies that the relevant wavelengths are of the order of a meter and greater; thus, fine details of the cavity geometry are unlikely to affect the behavior of aero-acoustical phenomena at these frequencies. In particular, of the components of the telescope structure, only the primary mirror is likely to play any significant role. In the present computations, the cavity geometry is simplified by the elimination of fine details and the telescope structure is entirely neglected. (The primary mirror will be introduced into the computations in later work.)

Second, Schmid et al. ${ }^{10}$ have shown that the frequency and shape of vibrational modes within the cavity are predicted nearly as well by a linear acoustic wave computation as they are by their DES computations. This indicates a full viscous solution with turbulence modeling in the cavity is unnecessary and computational time may be saved by solving only the Euler equations and, (probably most significantly) by not having to grid the cavity in order to resolve the shear layers at the walls.

The consequence of these two insights is we grid the geometrically simplified cavity and the farfield sparsely, adequate only for an Euler computation, and focus our grid points and full viscous/DES equations on the fuselage boundary layer and the free shear layer in the cavity doorway.

The initial, coarse, grid employed in this investigation is shown in Figure 1. The flow domain extends from just aft of the wing root to just forward of the rudder and consists only of the left side of the aircraft and the surrounding flow, from the fuselage to a cylinder of radius about $17.5 \mathrm{~m}$. The external portion of the flow is covered by a single block with 129 points in the axial $(x)$ direction, 97 points in the azimuthal direction and 97 grid points in the radial direction. The axial and azimuthal points are evenly spaced, except for mild clustering near the edges of the cavity doorway. The radial points are distributed so that the cell 
center of the first cell is approximately $1 \mathrm{~mm}$. above the fuselage surface and the next cell is $20 \%$ larger. This block is split into two blocks at the 37 th radial point in order to facilitate the mixed-equation solution described below.

The cavity doorway, including the lip in the aft portion, is grided with a single block with 49 points in each direction. Clustering in the axial and azimuthal directions are dictated by the external block; some clustering in the radial direction is done to avoid an abrupt change in cell size where this block fits against the external block.

The cavity itself is meshed with a block for the upper cavity (the half-cylinder nearest the cavity opening) and a block for the lower cavity (the half-cylinder furthest from cavity opening). In keeping with the discussion above, the cavity is meshed fairly coarsely, with the upper cavity block containing $55 \times 49 \times 49$ points and the lower cavity block containing $59 \times 29 \times 29$ points. A detail of the cavity and doorway meshing is shown in Figure 2.

As discussed in the following section, computations conducted with this grid suggested that increased shear-layer resolution in the streamwise and cross-stream directions would be desirable. A finer grid was thus constructed in which the exterior block was increased to $155 \times 145 \times 97$ grid points, the cavity doorway block was increased to $73 \times 73 \times 73$ grid points and the cavity blocks were increased to $83 \times 73 \times 73$ grid points and $89 \times 43 \times 43$ grid points. The cavity and doorway meshing for this grid is shown in Figure 3 .

The VULCAN code $\left(e . g .{ }^{11}\right)$ for structured grids was chosen for this computation due to its excellent performance in other projects and the fact that a number of DES models are available. It also permits the viscous and turbulence stresses to be turned on or off in individual grid blocks, permitting the computation to be set up so that the Euler equations are solved everywhere but in the fuselage boundary layer and the free shear layer in the cavity opening, where the full viscous/DES equations are solved.

Boundary conditions in the upper and lower cavity blocks are inviscid adiabiatic wall conditions, in keeping with the Euler-equation solution to be performed in these blocks. The third Euler block, the portion of the external block outside the fuselage boundary layer, employs freestream fixed boundary conditions on its inflow and far-field faces, symmetry conditions on the vertical faces and a subsonic outflow condition on the outflow face.

Adiabatic, viscous, wall boundary conditions with turbulence wall functions are used on the fuselage surface. Turbulence wall functions were not found to work well on the walls of the cavity doorway itself, especially on the lip at the aft of the doorway, so pure adiabatic viscous wall boundary conditions are used on the doorway walls.

Symmetry and outflow boundary conditions for the portion of the external block adjacent to the fuselage, containing the fuselage boundary layer, are the same as the outer portion. The inflow boundary condition is constructed from Prandtl's $u / u_{o}=(y / \delta)^{\frac{1}{n}}$ approximation for a turbulent boundary layer.

The computations are started by determining a steady-state solution, which is then used as the initial condition for the time-dependent solution. Dual time stepping is used, with a time step of $10^{-4}$ seconds. The residual was typically reduced by one order of magnitude in six or seven subiterations. This is a coarse time step, and one would generally prefer to see the residual reduced by at least two orders of magnitude, but experiments described below indicated that decreasing the time step or increasing the number of subiterations had only small effects on the results. Sticking with the coarse time step and smaller numbers of subiterations permitted more experiments to be conducted.

The DES scheme of Strelets ${ }^{1}$ is implemented in VULCAN and was employed in these computations. A value of $C_{D E S}=0.61$ was used for the constant modulating the cross-over from RANS behavior to LES behavior; this is the value determined by Strelets to give the correct behavior in a homogeneous-flow test case.

In these computations, the coarse grid was run on 64 processors and a full computation, from determining the steady-state solution used as the initial condition, through evolution of the time-dependent solution to a statistically steady state and ending with at least one second of the statistically steady state for computation of spectra, takes about 40 hours of wall-clock time, or about $2500 \mathrm{cpu}$-hrs. An attempt to decompose the grid in a manner suitable for 128 processors was abandoned when the decomposition process split the grid into computationally problematic blocks only a few cells thick. This could no doubt be remedied, but long-term use of this coarse grid is not planned and reaching an absolute minimum in wall-clock time was not a priority.

The finer grid was run on 128 processors and a full computation took about 34 hours of wall-clock time, or about $4300 \mathrm{cpu}-\mathrm{hrs}$. 


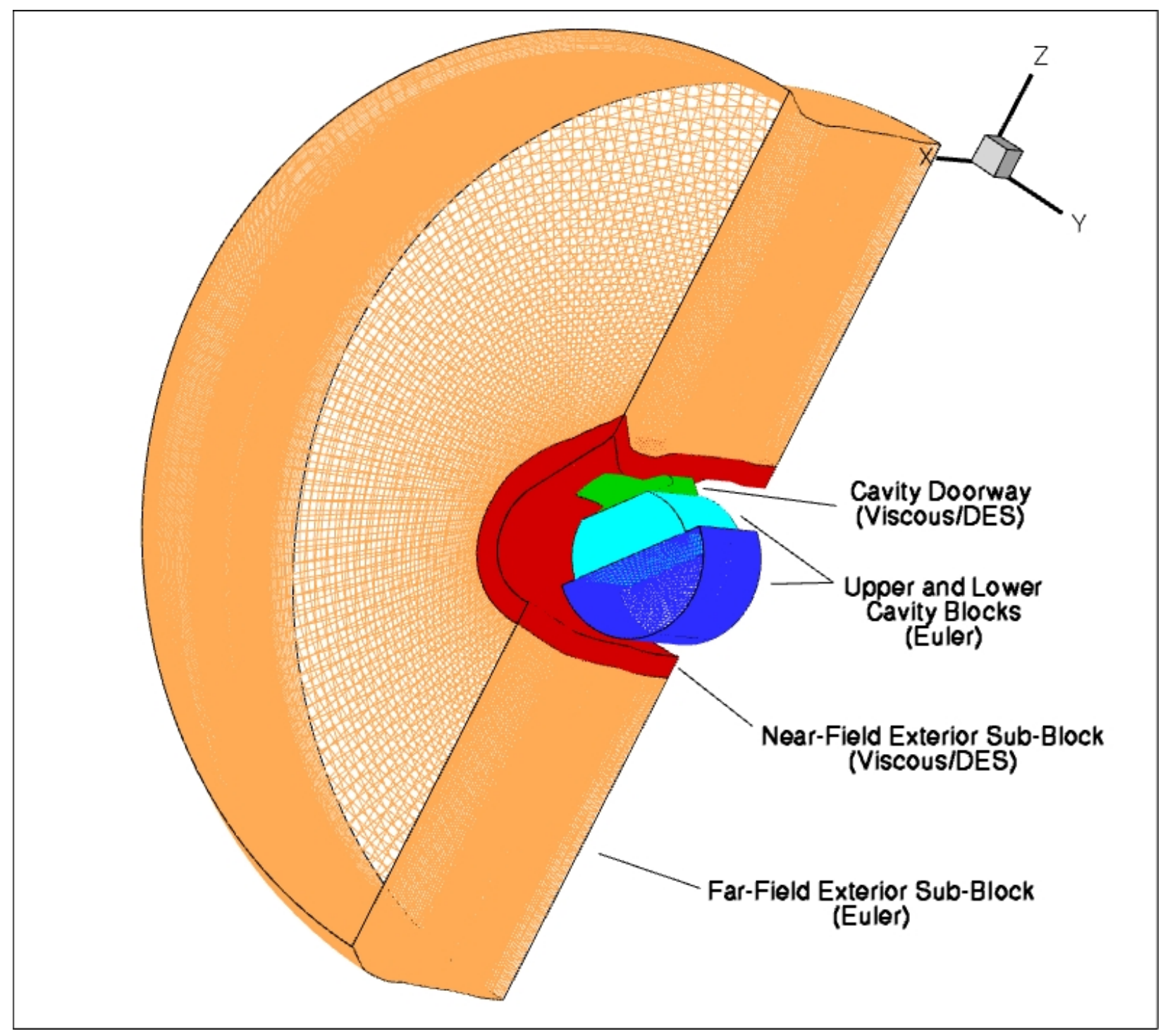

Figure 1. Coarse Mesh. (View from back of aircraft looking forward.)

\section{Results}

Wind tunnel tests have been conducted on a number of occasions on the SOFIA configuration (including $^{2,3}$ ). While the focus of much of the testing was on the effect of the cavity on steady-state aerodynamic force properties, time-domain acoustic data was also taken in several of the test programs at a variety of points in the cavity. As these provide the most detailed experimental information available about the time-dependent aspects of the cavity flow, this data is the primary means of validation of the present computations.

The wind-tunnel test reports describe qualitatively different low-speed and high speed resonance conditions. The computations reported here focus on two flight conditions, one at each of these resonance conditions. The lower-speed case is at a mach number of 0.60 and an altitude of 25,000 ft. The high speed case is at a mach number of 0.85 and an altitude of $40,000 \mathrm{ft}$.

Results of these computations for the $M=0.60$ case using the coarse grid described above are shown in Figure 4. The acoustic power spectrum measured at the center of the foreward cavity bulkhead is shown. [In the final paper, the experimental data will be included in these plots for direct comparison.] The computations yield the major peaks in the spectrum and give their frequency and amplitude with good accuracy. The computations with the coarse grid did tend to over-predict somewhat the pressure amplitudes 


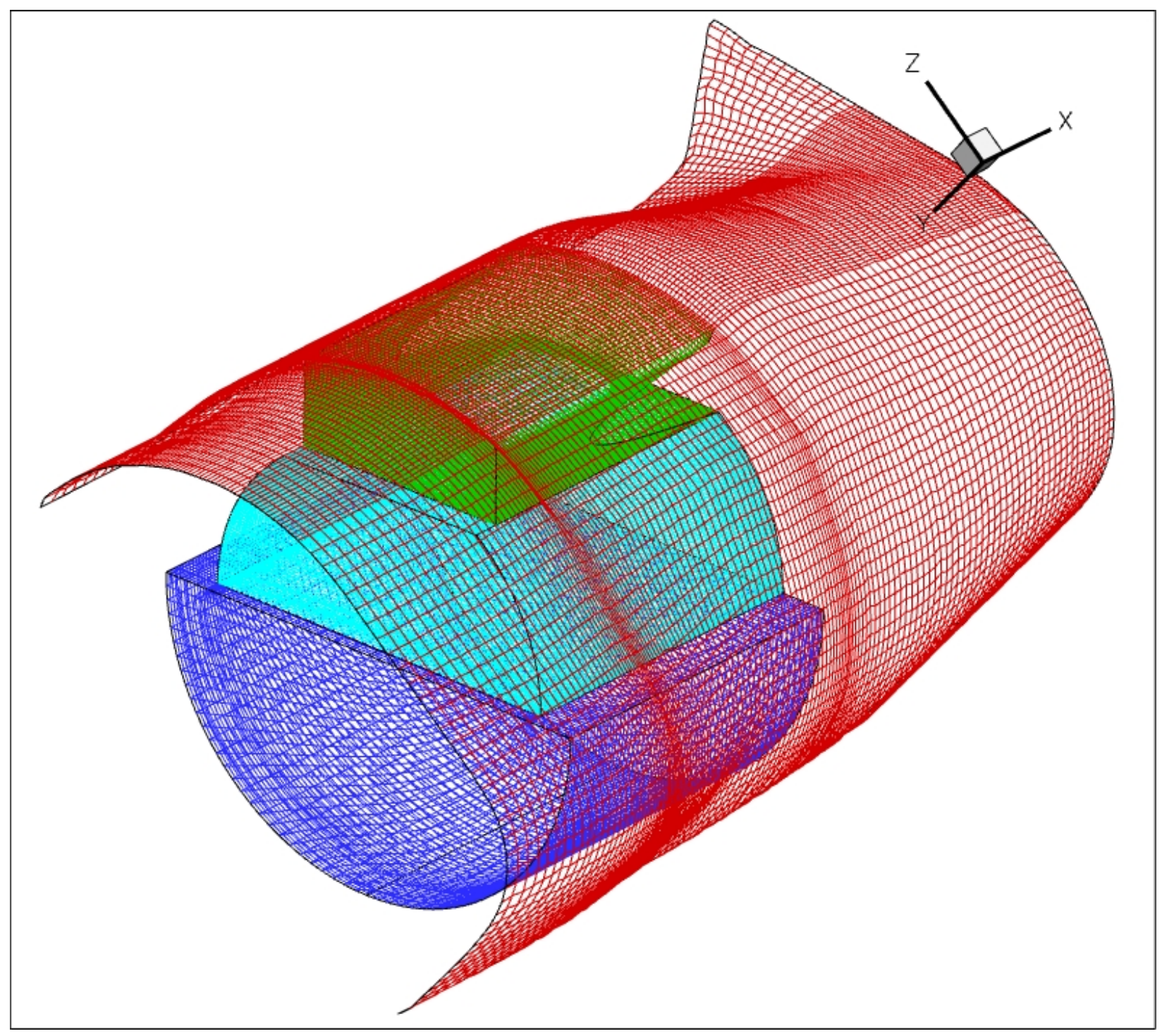

Figure 2. Close-Up of Cavity of Coarse Mesh. (View from left and front of aircraft looking back.)

at higher frequencies (above $100 \mathrm{~Hz}$ ). Experiments with reduced time steps and increased subiterations per time step were conducted to check that the pressure spectra were essentially unchanged. Use of the full viscous and turbulence equations in the entire flow domain was also investigated, with little change in the pressure spectra. (These latter computations were with the same grid as before, with inviscid (flow-tangency) boundary conditions at the walls of those domains formerly computed using the Euler equations, so wall shear layers were still not being resolved.)

Experiments with varying the boundary-layer thickness and turbulence levels at the upstream boundary were also conducted to get some understanding of the sensitivity of the cavity acoustics to these factors. Variation of pressure amplitudes with boundary-layer thickness has been reported in the literature and was seen here as well. Turbulence levels had an interestingly targeted effect: increased boundary-layer turbulence essentially eliminated some resonance peaks in the pressure spectrum, but left others unchanged. Full-aircraft RANS will be employed in future work to determine more accurately these upstream boundary conditions for specific flight conditions.

Finally, experiments with new, larger, grids were conducted to determine where grid refinement was required. In particular, the failure of increased temporal accuracy to improve the over-prediction of highfrequency amplitudes suggested better resolution of small-scale structures in the shear layers was required. The finer mesh described in the previous section was the result of these experiments, with the over-prediction 


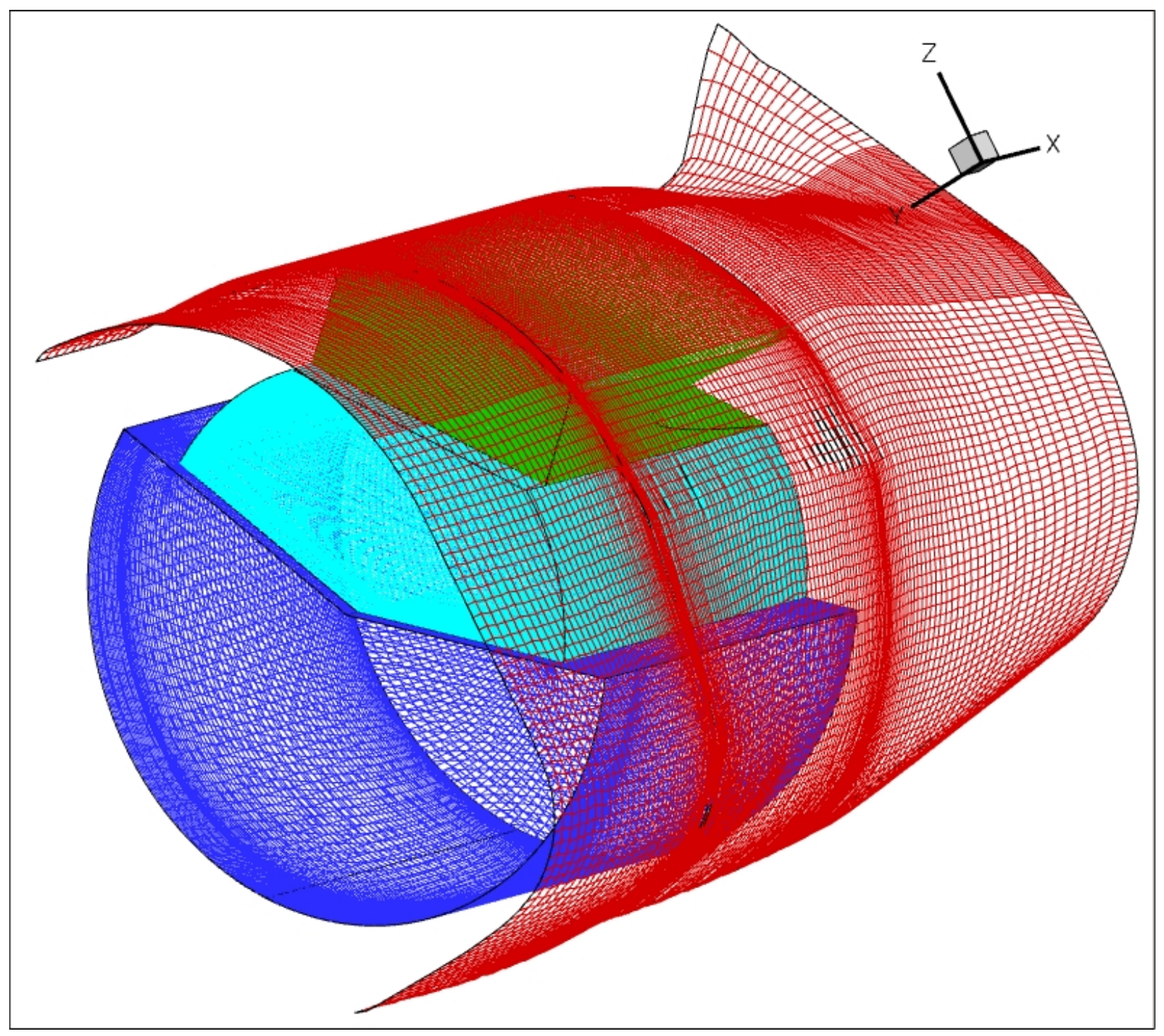

Figure 3. Close-Up of Cavity of Finer Mesh. (View from left and front of aircraft looking back.)

of frequencies above $100 \mathrm{~Hz}$ being fixed by improving the spanwise and streamwise grid resolution in and adjacent to the cavity opening. This is shown for the $M=0.60$ case in Figure 5 .

The results for the $M=0.85$ case using the coarse grid are shown in Figure 6. Although analysis is not quite complete, the conclusions drawn from the $M=0.60$ case generally seem to carry over to this case as well.

From the standpoint of parallel computation, no new ground is being broken in the present work. The parallelization of the computation is handled by VULCAN, a well-established, highly developed, parallel research code. As a result, the computations with the coarse grid, running on 64 processors, consistently maintained a computational efficiency of $96 \%-98 \%$. The finer grid was run on 128 processors and generally maintained the same level of computational efficiency, though at times it ran in the low to mid 90s.

\section{Conclusions}

The finer-mesh computation shown in Figure 5 took approximately 34 hours of wall-clock time on 128 processors. The corresponding coarse-grid computation took approximately 40 hours of wall-clock time on 128 processors. These times are significantly faster than those cited above for previous similar cavity computations. It has thus been demonstrated that a computation with simplified geometry and physics can 
capture the essential cavity resonance phenomena accurately enough to provide useful information much more rapidly than had previously been possible.

\{In the final paper, a fuller description of the results of the numerical experiments described in Section III will be given, as well as a discussion, based on the computational results, of the different flow mechanisms in the low-speed and high-speed resonance conditions.\}

\section{Acknowledgments}

Computer time for this work was provided by NAS.

\section{References}

${ }^{1}$ Strelets, M., "Detached Eddy Simulation of Massively Separated Flows," AIAA 2001-0879.

${ }^{2}$ Rose Engineering and Research, Inc., "SOFIA - Conceptual Door Design and Aero-Optics Wind Tunnel Test: SOFIA IV: Final Report"

${ }^{3}$ L3 Communications Integrated Systems, "Aerodynamic Data for the SOFIA Modification Model 747SP", Document Number: 96185007-001.

${ }^{4}$ Mendonca, F., Allen, R., de Charentenay, J. and Kirkham, D., "CFD Prediction of Narrowband and Broadband Cavity Acoustics at $\mathrm{M}=0.85 "$, AIAA 2003-3303.

${ }^{5}$ Basu, D., Hamed, A. and Das, K., "DES and Hybrid RANS/LES models for unsteady separated turbulent flow prediction", AIAA 2005-503.

${ }^{6}$ Tramel, R., Rock, S. and Ellis, J., "Comparison of Large Cavity Aeroacoustic Computations with Flight Test Results," AIAA 2005-2800.

${ }^{7}$ Schmid, S., Lutz, T. and Kramer, E., "Numerical Simulation of the Flow Field Around the Stratospheric Observatory For Infrared Astronomy", in Notes on Numerical Fluid Mechanics and Multidisciplinary Design, C. Tropea, S. Jakirlic, H. J. Heinemann, R. Henke and H. Honlinger, Eds., 96 Springer, Berlin, 2007.

${ }^{8}$ Schmid, S., Lutz, T., Kramer, E. and Kuhn, T., "Passive Control of the Flow of the Stratosphieric Observatory for Infrared", AIAA 2008-6717.

${ }^{9}$ Schmid, S., Lutz, T. and Kramer, E., "Simulation of the Flow Around the Stratospheric Observatory For Infrared Astronomy (SOFIA) Using URANS and DES," in Transactions of the Third Joint HLRB and KONWIHR Status and Result Workshop, Dec. 3-4, 2007, Leibniz Supercomputing Center, Garching/Munich, Germany. S. Wagner, M. Steinmetz, A. Bode, M. Brehm, Eds. 2009.

${ }^{10}$ Schmid, S., Lutz, T. and Kramer, E., "Simulation of the Unsteady Cavity Flow of the Stratospheric Observatory For Infrared Astronomy", in Proceedings of the IUTAM Symposium "Unsteady Separated Flows and their Control", Braza, M. and Hourigan, K., Eds., 2009.

${ }^{11}$ Litton, D. K., Edwards, J. R. and White, J. A., "Algorithmic Enhancements to the VULCAN Navier-Stokes Solver," AIAA 2003-3979. 


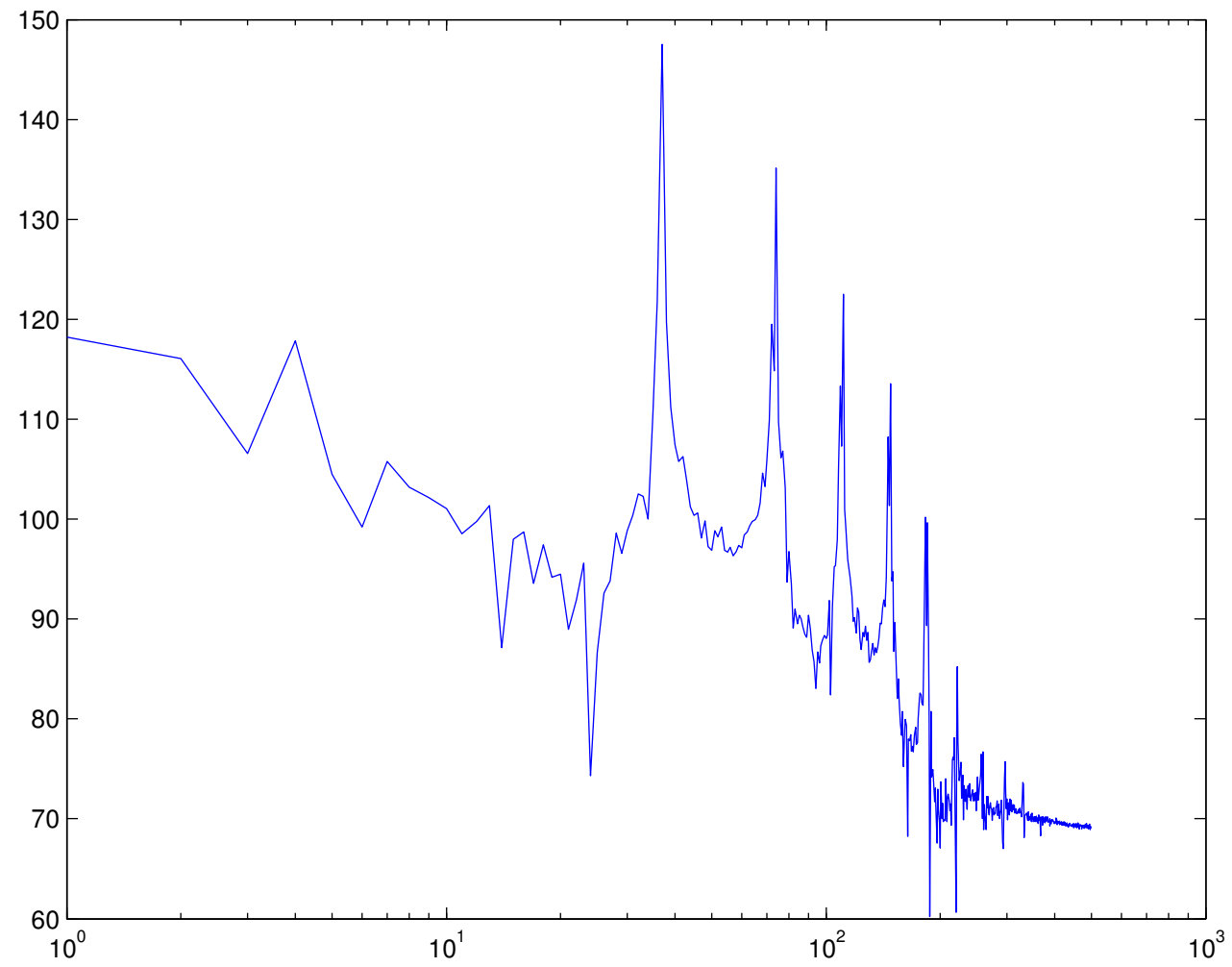

Figure 4. Acoustic Power (dB) Spectrum as function of Frequency (Hz) at forward bulkhead at M=0.6, coarse grid. 


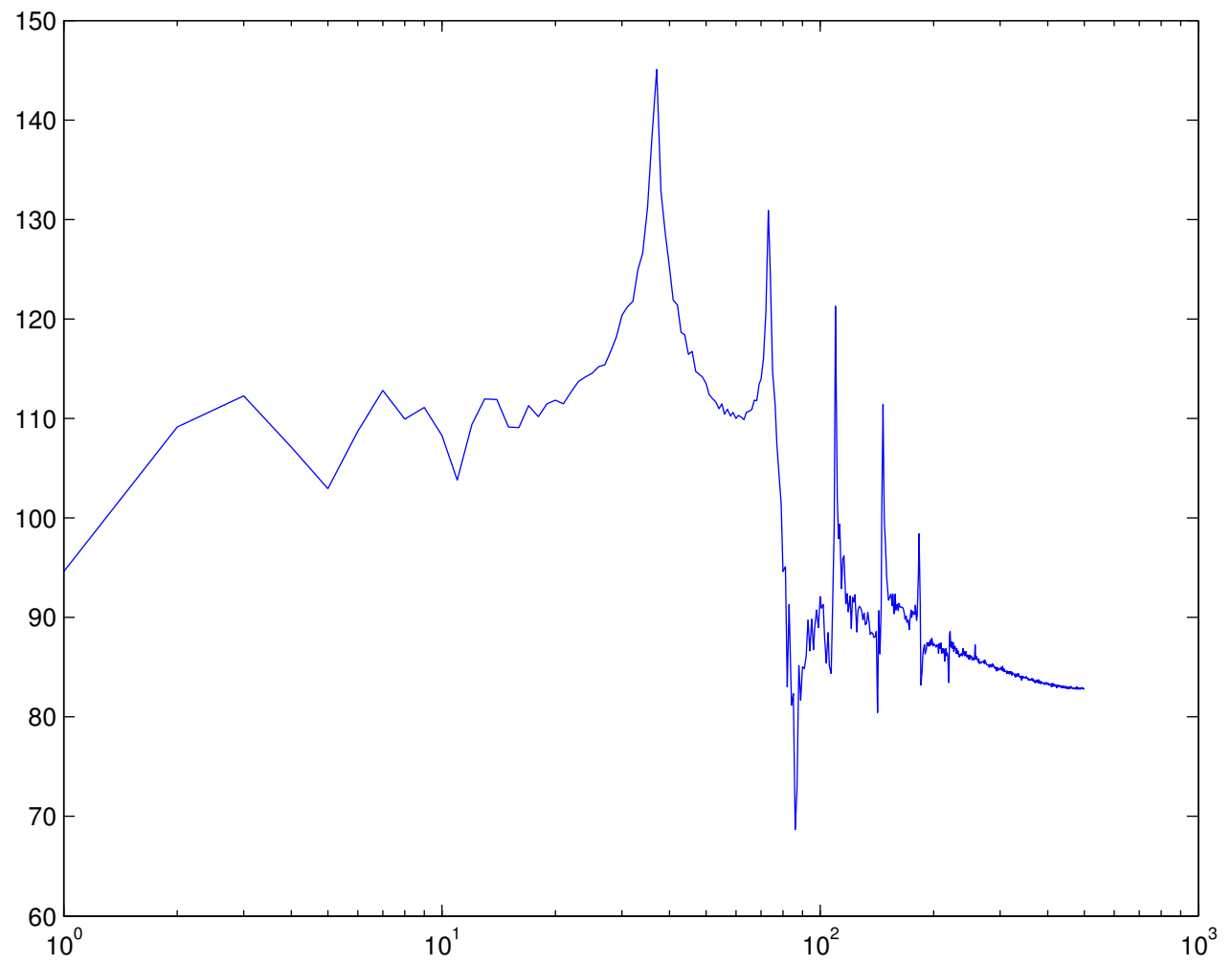

Figure 5. Acoustic Power (dB) Spectrum as function of Frequency (Hz) at forward bulkhead at M=0.6, finer grid.

$$
9 \text { of } 10
$$




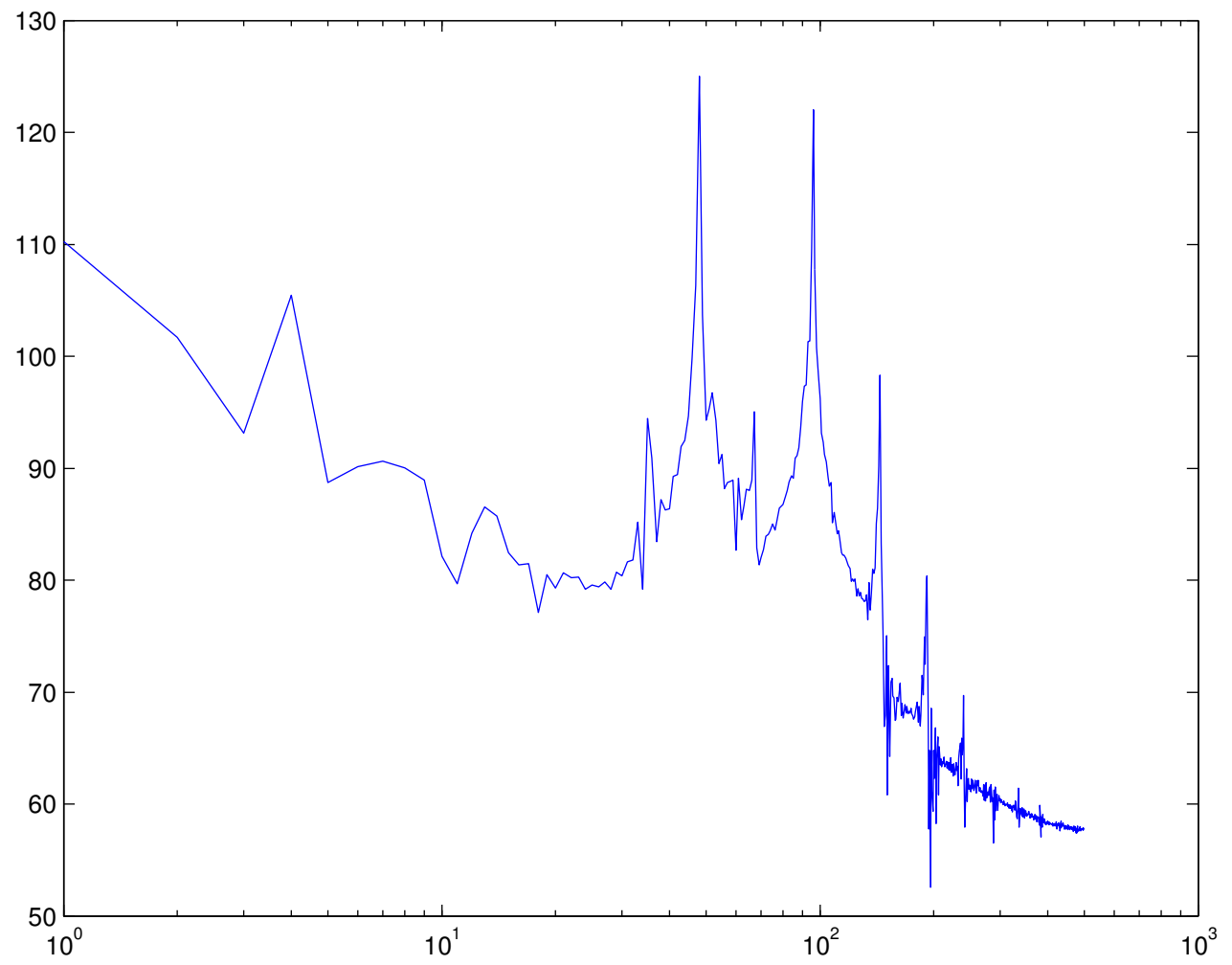

Figure 6. Acoustic Power (dB) Spectrum as function of Frequency (Hz) at forward bulkhead at M=0.85, coarse grid. 